\title{
Outer irregular satellites of the planets and their relationship with asteroids, comets and Kuiper Belt objects
}

\author{
Scott S. Sheppard ${ }^{1}$ \\ ${ }^{1}$ Department of Terrestrial Magnetism, Carnegie Institution of Washington, Washington, DC \\ 20015, USA \\ email: sheppard@dtm.ciw.edu
}

\begin{abstract}
Outer satellites of the planets have distant, eccentric orbits that can be highly inclined or even retrograde relative to the equatorial planes of their planets. These irregular orbits cannot have formed by circumplanetary accretion and are likely products of early capture from heliocentric orbit. The irregular satellites may be the only small bodies remaining which are still relatively near their formation locations within the giant planet region. The study of the irregular satellites provides a unique window on processes operating in the young solar system and allows us to probe possible planet formation mechanisms and the composition of the solar nebula between the rocky objects in the main asteroid belt and the very volatile rich objects in the Kuiper Belt. The gas and ice giant planets all appear to have very similar irregular satellite systems irrespective of their mass or formation timescales and mechanisms. Water ice has been detected on some of the outer satellites of Saturn and Neptune whereas none has been observed on Jupiter's outer satellites.
\end{abstract}

Keywords. planets and satellites: general, Kuiper Belt, minor planets, asteroids, comets: general, solar system: formation

\section{Introduction}

Satellites are stable in the region called the Hill sphere in which the planet, rather than the sun, dominates the motion of the object (Henon 1970). The Hill sphere radius of a planet is defined as

$$
r_{H}=a_{p}\left[\frac{m_{p}}{3 M_{\odot}}\right]^{1 / 3}
$$

where $a_{p}$ and $m_{p}$ are the semi-major axis and mass of the planet and $M_{\odot}$ is the mass of the sun. Table 1 shows the sizes of each giant planet's Hill sphere as seen from the Earth at opposition.

Most planetary satellites can be classified into one of two categories: regular or irregular (Kuiper (1956); Peale (1999)).

The regular satellites are within about $0.05 r_{H}$ and have nearly circular, prograde orbits with low inclinations near the equator of the planet. These satellites are thought to have formed around their respective planets through circumplanetary accretion, similar to how the planets formed in the circumstellar disk around the sun. The regular satellites can be subdivided into two types: classical regulars and collisional shards (Burns 1986).

The classical regular satellites are large (several hundred to thousands of kilometers in size) and have evenly spaced orbits. The regular collisional shards are small (less than a few hundred kilometers) and are believed to have once been larger satellites but have been shattered or tidally disrupted over their lifetimes. These shards are usually very near 
Table 1. Irregular Satellites of the Planets

\begin{tabular}{lcccccc}
\hline Planet & $\begin{array}{c}\operatorname{Irr}^{a} \\
(\#)\end{array}$ & $\begin{array}{c}m_{p} \\
\left(10^{25} \mathrm{~kg}\right)\end{array}$ & $\begin{array}{c}r_{\min }{ }^{b} \\
(\mathrm{~km})\end{array}$ & $\begin{array}{c}a_{\mathrm{crit}} \\
\left(10^{6} \mathrm{~km}\right)\end{array}$ & $\begin{array}{c}r_{H}{ }^{c} \\
(\mathrm{deg})\end{array}$ & $\begin{array}{c}r_{H} \\
\left(10^{7} \mathrm{~km}\right)\end{array}$ \\
\hline Mars & 0 & 0.06 & 0.1 & & 0.8 & 0.1 \\
Jupiter & 55 & 190 & 1.5 & 6.6 & 4.7 & 5.1 \\
Saturn & 26 & 57 & 3 & 5.7 & 3.0 & 6.9 \\
Uranus & 9 & 9 & 7 & 2.9 & 1.5 & 7.3 \\
Neptune & $6(7)$ & 10 & 16 & 3.8 & 1.5 & 11.6 \\
\hline
\end{tabular}

a) The number of known irregular satellites.

b) Minimum radius that current outer satellite searches would have detected to date.

c) Size of the Hill sphere as seen from Earth at opposition.

the planet where tidal forces and meteor fluxes are very high. Many collisional shards are associated with known planetary rings.

In contrast, the irregular satellites have semi-major axes $>0.05 r_{H}$ with apocenters up to $0.65 r_{H}$ (Figure 1). Irregular satellites have eccentric orbits that are usually highly inclined and distant from the planet. They can have both prograde and retrograde orbits. The irregular satellites can not have formed around their respective planet with their current orbits and are likely the product of early capture from heliocentric orbits (Kuiper 1956).

Orbital characteristics displaying strong capture signatures instead of in situ formation around the planet is often used to define an irregular satellite. Throughout this work we will use a more strict definition. We follow others and define irregular satellites as those satellites which are far enough from their parent planet that the precession of their orbital plane is primarily controlled by the sun instead of the planet's oblateness. In other words, the satellite's inclination is fixed relative to the planet's orbit plane instead of the planet's equator. In practice this means any satellite with a semi-major axis more distant than the critical semi-major axis (Burns 1986), $a_{\text {crit }} \sim\left(2 J_{2} r_{p}^{2} a_{p}^{3} m_{p} / M_{\odot}\right)^{1 / 5}$, is an irregular satellite (Table 1 ). Here $J_{2}$ is the planet's second gravitational harmonic coefficient and $r_{p}$ is the planet's equatorial radius. Figures 1 and 2 show the orbital characteristics of the known irregular satellites. In these figures all known regular satellites would fall very near the origin.

Almost all known planetary satellites fall into one of the three types mentioned above. A few exceptions do exist. The formation of the Earth's Moon is best explained through a collision between a Mars sized body and the young Earth (see Canup \& Asphaug 2001 and references therein). Mars' two small satellites Phobos and Deimos resemble regular collisional shards, but some have suggested that they may be captured bodies similar to the irregular satellites of the giant planets (Burns 1992). No outer irregular satellites of Mars are known to exist (Sheppard \& Jewitt 2004). Both Neptune's Triton and Saturn's Iapetus have at times been considered irregular satellites. These two objects stand out because both Triton and Iapetus are about ten times larger than any other known irregular satellite. Triton has all the characteristics of a regular satellite except that its orbit is retrograde. The best explanation for a retrograde orbit is through capture. Iapetus also has all the characteristics of a regular satellite but its inclination of 7 degrees is significantly larger than any other known regular satellite. Even so, this inclination is not as high as the vast majority of irregular satellites. Iapetus' relatively large inclination is probably because it is the most distant regular satellite of Saturn. At this distance the circumSaturnian nebula was probably of low density which dissipated quickly. These factors would have significantly slowed or stopped the process of orbital evolution. 


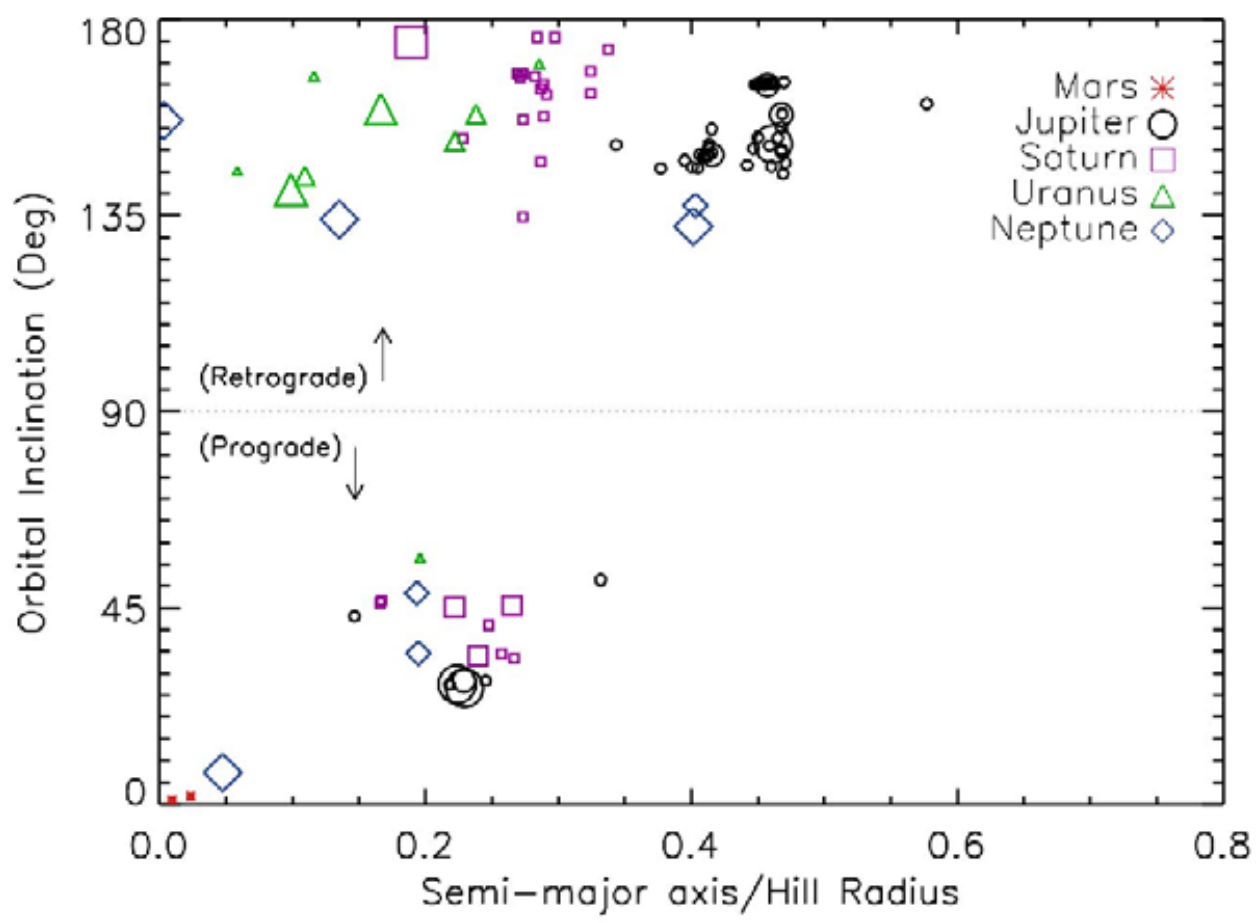

Figure 1. All 96 Known irregular satellites of the giant planets. The horizontal axis is the ratio of the satellites semi-major axis to the respective planet's Hill radius. The vertical axis is the inclination of the satellite to the orbital plane of the planet. The size of the symbol represents the radius of the object: Large symbol $r>25 \mathrm{~km}$, medium symbol $25>r>10 \mathrm{~km}$, and small symbol $r<10 \mathrm{~km}$. Neptune's Triton can be seen in the upper left of the figure while Nereid is near the lower left. Mars' two satellites are plotted for comparison. All 53 known regular satellites would fall near the origin of this plot. [Modified from Sheppard et al. 2005]

Finally, Neptune's outer satellite Nereid is usually considered an irregular satellite but its relatively small semi-major axis and low inclination yet exceptionally large eccentricity suggest it may be a perturbed regular satellite, perhaps from Triton's capture (Goldreich et al. 1989; Cuk \& Gladman 2005; Sheppard et al. 2006).

\section{Irregular Satellite Discovery}

Irregular satellite discovery requires large fields of view because of the large planetary Hill spheres. Sensitivity is needed because the majority of irregular satellites are small (radii $<50 \mathrm{~km}$ ) and therefore faint. With the use of large field-of-view photographic plates around the end of the 1800's the first distinctive irregular satellites were discovered (Figure 3). In 1898 the largest irregular satellite of Saturn, Phoebe (radius $\sim 60 \mathrm{~km}$ ), was discovered and in 1904 the largest irregular of Jupiter, Himalia (radius $\sim 92 \mathrm{~km}$ ), was discovered (see Kuiper 1961 for a review of early photographic surveys).

Until 1997 only ten or eleven irregular satellites were known and the last discovered irregular satellite was in 1975 on photographic plates (Kowal et al. 1975). Since 1997 eighty-six irregular satellites have been discovered around the giant planets (Gladman et al. 1998;2000;2001; Sheppard \& Jewitt 2003; Holman et al. 2004; Kavelaars et al. 2004; Sheppard et al. 2005;2006). Jupiter's retinue of irregular satellites has increased from 8 to 55, Saturn's from 1 to 26, Uranus' from 0 to 9 and Neptune's from 1 to 6 (or seven if 


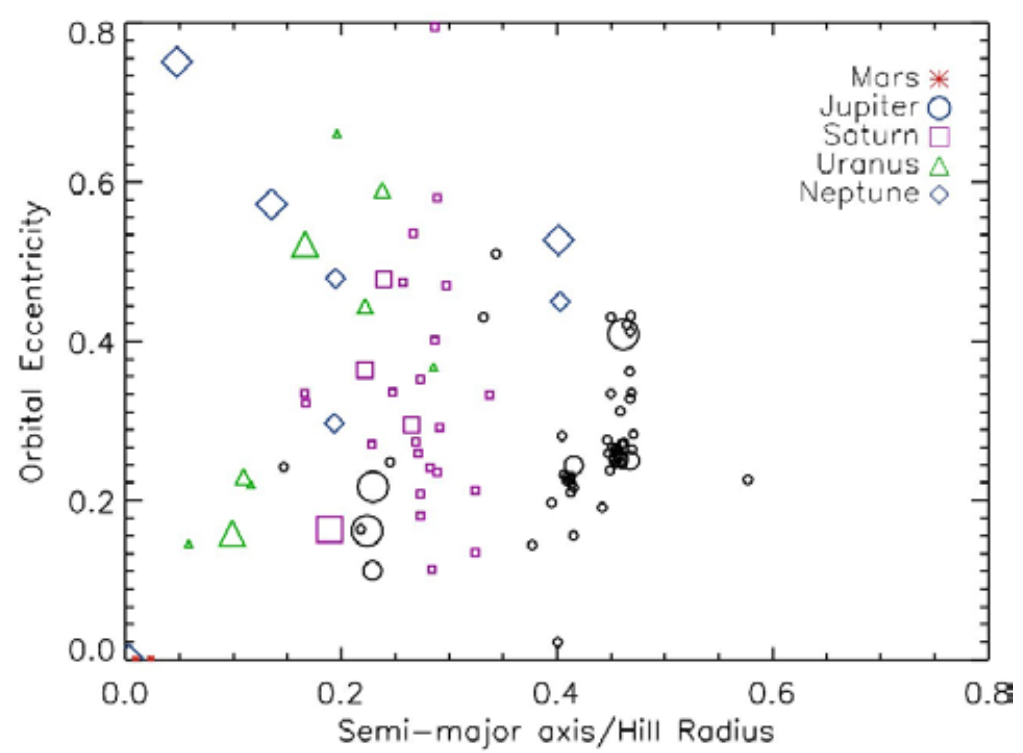

Figure 2. All 96 Known irregular satellites of the giant planets. The horizontal axis is the ratio of the satellites semi-major axis to the respective planet's Hill radius. The vertical axis is the orbital eccentricity. The size of the symbol represents the radius of the object: Large symbol $r>25 \mathrm{~km}$, medium symbol $25>r>10 \mathrm{~km}$, and small symbol $r<10 \mathrm{~km}$. Again, all $53 \mathrm{known}$ regular satellites would fall near the origin of this plot, where Triton and Mars' satellites are located. [Modified from Sheppard et al. 2005]

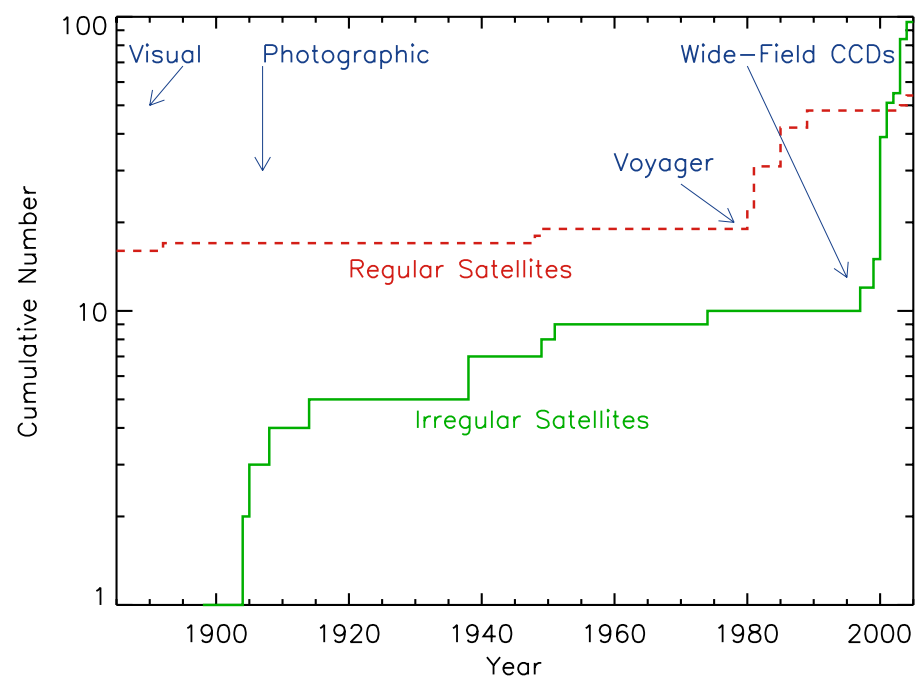

Figure 3. The number of irregular and regular satellites discovered since the late 1800's. Key technological advances which resulted in a jump in discoveries are listed.

including Triton). Table 1 shows information about the current irregular satellite systems around the giant planets.

The number of known irregular satellites (96 as of November 2005) have recently surpassed the number of known regular satellites (Figure 3). The main reason is new 


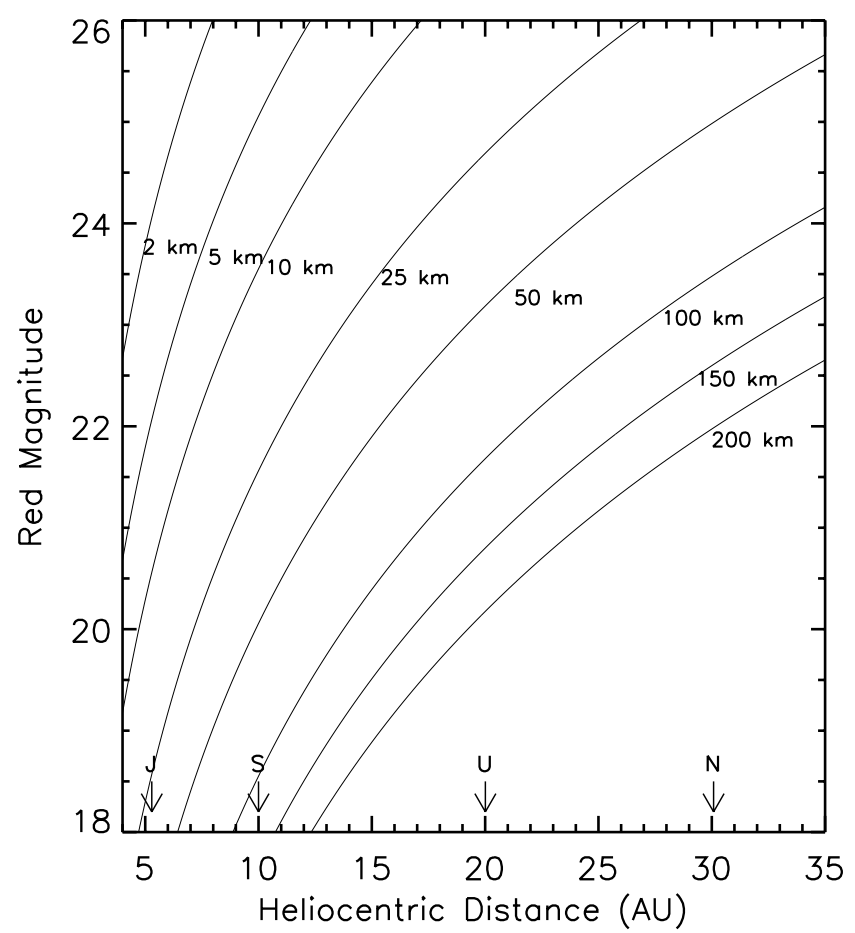

Figure 4. The distances of the planets versus the observable small body population diameter for a given red magnitude assuming an albedo of 0.04 . Jupiter's closer proximity allows us to probe the smallest satellites.

technology. The recent development of sensitive, large scale CCD detectors has allowed these faint outer planetary satellites to be discovered. Because of the proximity of Jupiter (Figure 4), it currently has the largest irregular satellite population (Sheppard and Jewitt 2003).

\section{Capture of Irregular Satellites}

Only the four giant planets have known irregular satellite populations (Figure 1). The likely reason is that the capture process requires something that the terrestrial planets did not have. Capture of a heliocentric orbiting object is likely only if the object approaches the planet near its Lagrangian points and has an orbital velocity within about $1 \%$ that of the planet. Objects may temporarily orbit a planet (i.e. Shoemaker-Levy 9) but because of the reversibility of Newton's equations of motion some form of energy dissipation is required to permanently capture a body. Without dissipation the object will be lost in less than a few hundred years (Everhart 1973; Heppenheimer \& Porco 1977). In the present epoch a planet has no known efficient mechanism to permanently capture satellites (Figure 5).

Kuiper (1956) first suggested that the irregular satellites were originally regular satellites which escaped from the planet's Hill sphere to heliocentric orbit because of the decreasing mass of the planet. These "lost" satellites would have similar orbits as the parent planet. Eventually the satellite would pass near the planet and be slowed down from the mass escaping from the planet. The satellite would thus be captured in an 


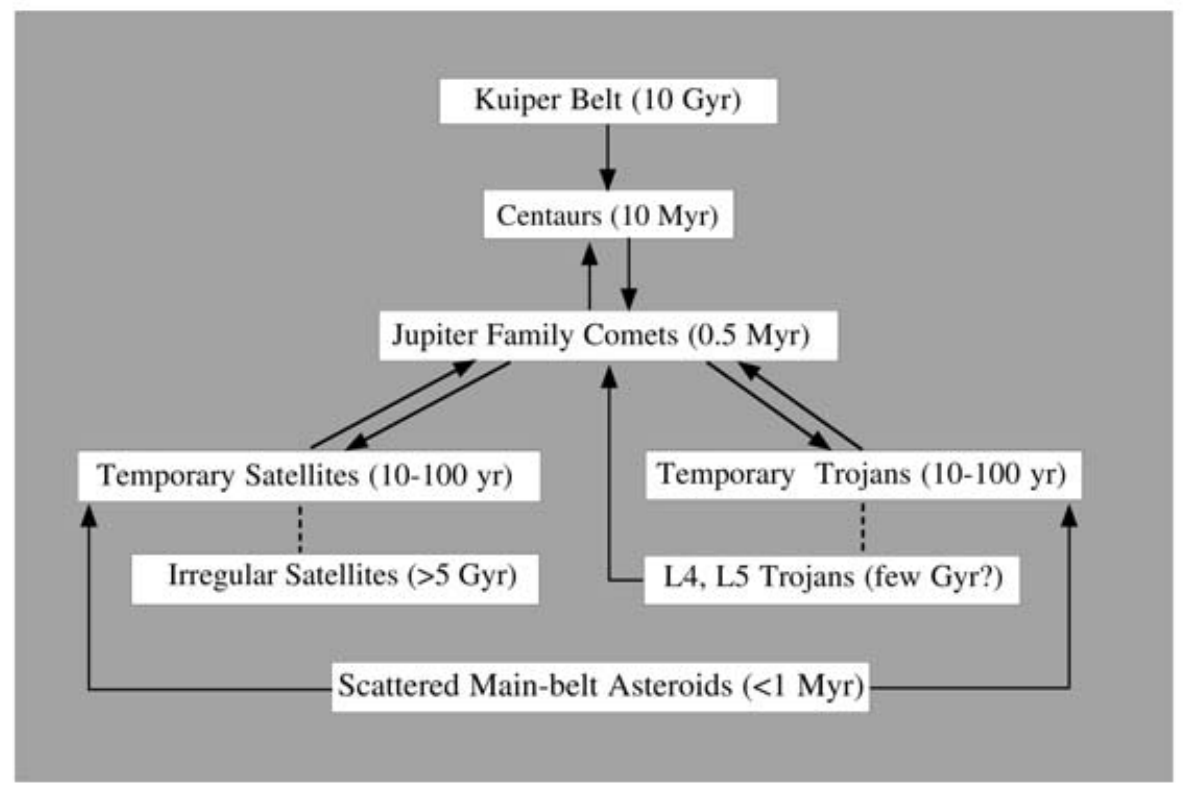

Figure 5. Interrelations among the small body populations in the solar system. Solid arrows denote established dynamical pathways. Dashed lines show pathways which currently have no known energy dissipation source and thus can not lead to permanent capture but only temporary capture. During the planet formation epoch such pathways may have existed. Numbers in parentheses indicate the approximate dynamical lifetimes of the different populations. (Figure from Jewitt et al. (2004))

"irregular" type orbit. It is now believed that the giant planets never lost significant amounts of mass and thus irregular satellites are unlikely to be escaped regular satellites.

The dissipation of energy through tidal interactions between the planet and irregular satellites is not significant for such small objects at such large distances (Pollack et al. 1979). The creation of irregular satellites from explosions of the outer portions of the massive ice envelopes of the large regular satellites from saturation by electrolysis seems unlikely and no observational evidence supports such explosions on the regular satellites (Agafonova \& Drobyshevski 1984).

Three viable mechanisms have been proposed for irregular satellite capture. Satellite capture could have occurred efficiently towards the end of the planet formation epoch due to gas drag from an extended planetary atmosphere (Kuiper 1956; Pollack, Burns \& Tauber 1979), the enlargement of the Hill sphere caused by the planet's mass growth (Heppenheimer \& Porco 1977) and/or higher collisional or collisionless interaction probabilities with nearby small bodies (Colombo \& Franklin 1971; Tsui 2000). Below we discuss each of these in more detail.

\subsection{Capture by Gas Drag}

During early planet formation the giant planets likely had primordial circumplanetary nebulae (Pollack et al. 1979; Cuk \& Burns 2004). An object passing through this gas and dust near a planet would have experienced gas drag. In order to significantly slow 
an object for capture it would need to encounter about its own mass within the nebula. Conditions at the distances of the irregular satellites are unknown, but rough estimates suggest that if the object was larger than a few hundred kilometers it would not have been significantly affected. If the object was very small it would have been highly slowed and would have spiraled into the planet. If the object was just the right size (a few $\mathrm{km}$ to a few hundred kilometers) is would have experienced just enough gas drag to be captured (Pollack et al. 1979). Hydrodynamical collapse of the primordial planetary nebula would have to occur within a few thousand years of capture in order for the satellites to not experience significant orbital evolution and eventually spiral into the planet from gas drag. In this scenario the current irregular satellites are only the last few captured bodies which did not have time to spiral into the planet. Retrograde objects would have experienced larger gas drag during their time within the nebula and thus their orbits should be more modified toward smaller eccentricities, inclinations and semimajor axes. Observations currently show that both the progrades and retrogrades have similar modification. Gas drag would also allow for larger objects to be captured closer to the planet since the nebula would be more dense there. In the action of gas drag smaller irregular satellites should have their orbits evolve faster and should have been preferentially removed. No size versus orbital characteristics are observed for any of the irregular satellites of the planets.

\subsection{Pull-down Capture}

Another way an object can become permanently captured is if the planet's mass increased or the Sun's mass decreased while the object was temporarily captured, called pull-down capture (Heppenheimer \& Porco 1977). Either of these scenarios would cause the Hill sphere of the planet to increase making it impossible for the object to escape with its current energy. Again, the enlargement of the Hill sphere would have to happen over a short timescale. Likely mass changes of the Sun or the planet would need to be greater than about $40 \%$ over a few thousand years (Pollack et al. 1976). The Hill sphere of the planet would also increase if the planet migrated significantly away from the sun (Brunini 1995). This mechanism is not a likely cause of permanent capture because the large migrations required to make temporary capture permanent within a few thousand years would severely disrupt any satellite systems (Beauge et al. 2002).

\subsection{Capture Through Collisional or Collisionless Interactions}

Finally, a third well identified mechanism of capture could be from the collision or collisionless interaction of two small bodies within the Hill sphere of the planet (Colombo \& Franklin; Tsui 2000; Astakhov et al. 2003; Funato et al. 2004; Agnor \& Hamilton 2004). This could occur as asteroid-asteroid or asteroid-satellite encounters. These encounters could dissipate the required amount of energy from one or both of the objects for permanent capture. This mechanism for capture would operate much more efficiently during the early solar system when many more small bodies where passing near the planets. An interesting point of this capture mechanism is that it would be fairly independent of the mass or formation scenario of the planet and mostly depend on the size of the Hill sphere and number of passing bodies.

\section{Dynamics of Irregular Satellites}

The known irregular satellites are stable over the age of the Solar System though strongly influenced by solar and planetary perturbations (Henon 1970; Carruba et al. 2002; Nesvorny et al. 2003). The perturbations are most intense when the satellite is near 
apoapsis. High inclination orbits have been found through numerical simulations to be unstable due to solar perturbations (Carruba et al. 2002; Nesvorny et al. 2003). Satellites with inclinations between $50<i<130$ degrees slowly have their orbits stretched making them obtain very high eccentricities. The high eccentricities are obtained in $10^{7}-10^{9}$ years and cause the satellite to eventually be lost from the system either through exiting the Hill sphere or colliding with a regular satellite or the planet.

A number of irregular satellites have been found to be in orbital resonances with their planet. These resonances protect the satellites from strong solar perturbations. The two main types of resonance found to date are Kozai resonances and secular resonances (Kozai 1962; Carruba et al. 2002; Nesvorny et al. 2003). The irregular satellites known or suspected of being in resonances are Jupiter's irregular satellites Sinope, Pasiphae, Euporie (S/2001 J10), S/2003 J18 and Carpo (S/2003 J20) and Saturn's irregular satellites Siarnaq (S/2000 S3), Kiviuq (S/2000 S5) and Ijiraq (S/2000 S6) and Uranus' Stephano (Saha \& Tremaine 1993; Whipple \& Shelus 1993; Nesvorny et al. 2003; Cuk \& Burns 2004; R. Jacobson person communication). These resonances occupy a very small amount of orbital parameter space. The evolution of satellites into these resonances implies some sort of slow dissipation mechanism which allowed the satellites to acquire the resonances and not jump over them. This could be obtained from weak gas drag, a small increase in the planet's mass or a slow migration of the planet.

From numerical and analytical work it has been found that retrograde orbits are more stable than prograde orbits over large time-scales (Moulton 1914; Hunter 1967; Henon 1970; Hamilton \& Krivov 1997). Analytically the retrogrades may be stable up to distances of $\sim 0.7 r_{H}$ while progrades are only stable up to $0.5 r_{H}$ (Hamilton \& Krivov 1997). This is consistent with known orbits of retrogrades and progrades to date. Known retrograde (prograde) irregular satellites have semi-major axes out to $\sim 0.47 r_{H}\left(\sim 0.33 r_{H}\right)$ and have apocenters up to $\sim 0.65 r_{H}\left(\sim 0.47 r_{H}\right)$.

Many of the irregular satellites have been found to show dynamical groupings (Gladman et al. 2001; Sheppard \& Jewitt 2003; Nesvorny et al. 2003). At Jupiter the dynamical groupings are well observed in semi-major axis and inclination phase space (Figure 1) and are probably similar to families found in the main belt asteroids which are created when a larger parent body is disrupted into several smaller daughter fragments. The irregular satellites at the other giant planets are mostly grouped in inclination phase space and not in semi-major axis phase space. It would be unlikely that a fragmented body would create daughter bodies with such significantly different semi-major axes. This inclination clustering may just be because of resonance effects or that these particular inclinations are more stable. Still, there do appear to be some irregular satellites at Saturn, Uranus and Neptune that do cluster in semi-major axis and inclination phase space like those seen at Jupiter but these groups are not well populated. Further satellites in these putative dynamical families may be observed when smaller satellites are able to be discovered in the future.

Fragmentation of the parent satellites could be caused by impact with interplanetary projectiles (principally comets) or by collision with other satellites. Collisions with comets are improbable in the current solar system but during the heavy bombardment era nearly 4.5 billion years ago they would have been highly probable (Sheppard \& Jewitt 2003). Large populations of now defunct satellites could also have been a collisional source in creating the observed satellite groupings (Nesvorny et al. 2004). No size versus orbital property correlations are seen in the groupings which suggest breakup occurred after any significant amounts of gas were left. 


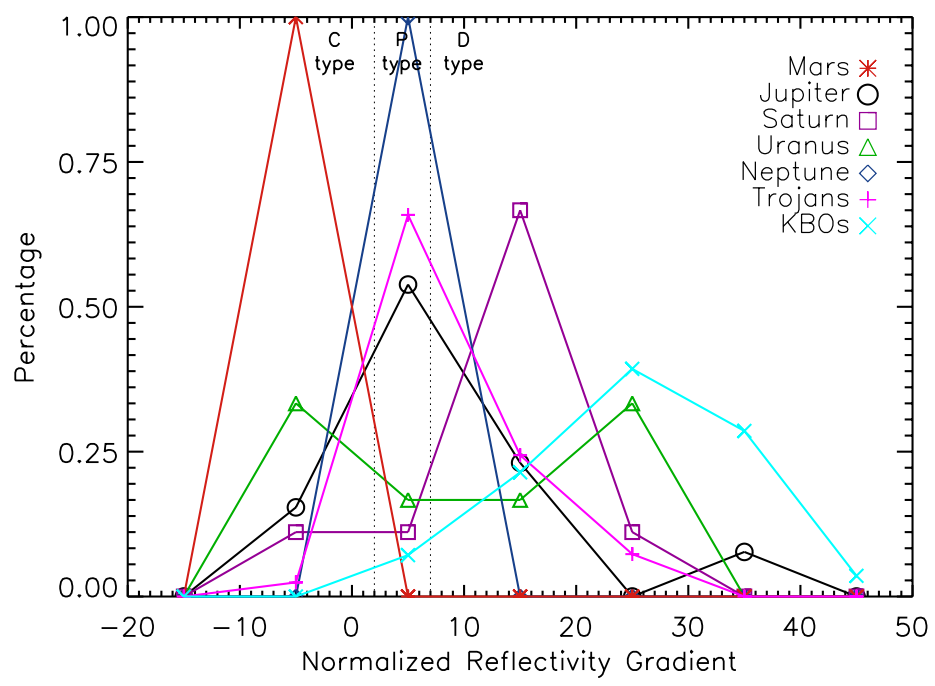

Figure 6. The colors of the irregular satellites of Jupiter, Saturn, Uranus and Neptune compared to the KBOs, Trojans and Martian satellites. The Jupiter irregular satellites are fairly neutral in color and very similar to the nearby Jupiter Trojans. Saturn's irregulars are significantly redder than Jupiter's but do not reach the extreme red colors seen in the KBOs. Uranus' irregular satellites are very diverse in color with some being the bluest known while others are the reddest known irregular satellites. Only two of Neptune's irregulars have measured colors and not much can yet be said except they don't show the very red colors seen in the Kuiper Belt. The general linear colors of the C, P and D-type asteroids are shown for reference (Dahlgren \& Lagerkvist 1995). Irregular satellite colors are from Grav et al. 2003; 2004a.

The detection of dust in bound orbits about Jupiter in the outer Jupiter system from the Galileo spacecraft is attributed to high velocity impacts of interplanetary micrometeoroids into the atmosphereless outer satellites (Krivov et al. 2002). The micron sized dust is in prograde and retrograde orbits with a number density $\left(10 \mathrm{~km}^{-3}\right)$ about ten times larger than in the local interplanetary medium.

\section{Physical Properties of Irregular Satellites}

Most of the space in the giant planet region of the solar system is devoid of objects which make irregular satellites one of the only dynamical clues as to what affected most of the mass in the solar system 4.5 billion years ago. Irregular satellites were likely asteroids or comets in heliocentric orbit which did not get ejected into the Oort cloud or incorporated in the planets. They may be some of the only small bodies remaining which are still relatively near their formation locations within the giant planet region. The irregular satellite reservoirs lie between the main belt of asteroids and Kuiper Belt which makes them a key to showing us the complex transition between rocky objects in the main asteroid belt and the expected very volatile rich objects in the Kuiper Belt.

\subsection{Visible and Infrared Colors}

Colors of the irregular satellites are neutral to moderately red (Tholen \& Zellner 1984; Luu 1991; Rettig et al. 2001; Maris et al. 2001; Grav et al. 2003; 2004a). Most do not show the very red material found in the distant Kuiper Belt (Figures 6 and 7). The Jupiter irregular satellite colors are very similar to the $\mathrm{C}, \mathrm{P}$ and $\mathrm{D}$-type carbonaceous outer main 


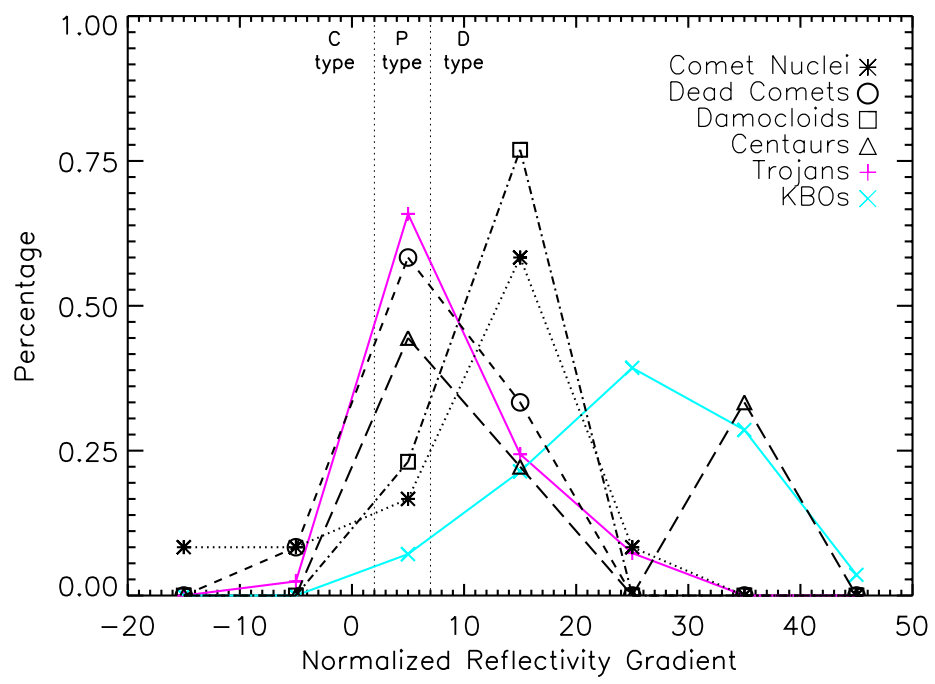

Figure 7. Same as Figure 6 except this plot shows the colors of the comet nuclei, dead comet candidates, Damocloids, Centaurs, Trojans and KBOs. It is plotted as a separate graph from Figure 6 to avoid confusion between the many different types of objects. Jupiter's irregulars are similar in color to the dead comets and some of the Centaurs. Saturn's irregulars are similar in color to the Damocloids and active comet nuclei. Comet nuclei and dead comet colors are from Jewitt (2002) and references therein. Centaur and KBO colors are from Barucci et al. (2001); Peixinho et al. (2001); Jewitt \& Luu (2001) and references therein. Damocloid colors are from Jewitt (2005) and references therein.

belt asteroids (Degewij et al. 1980) as well as to the Jupiter Trojans and dead comets. Colors of the Jupiter irregular satellite dynamical groupings are consistent with, but do not prove, the notion that each group originated from a single undifferentiated parent body. Optical colors of the 8 brightest outer satellites of Jupiter show that the prograde group appears redder and more tightly clustered in color space than the retrograde irregulars (Rettig et al. 2001; Grav et al. 2003). Near-infrared colors recently obtained of the brighter satellites agree with this scenario and that the Jupiter irregular's colors are consistent with D and C-type asteroids (Sykes et al. 2000; Grav et al. 2004b).

The Saturn irregular satellites are redder than Jupiter's but still do not show the very red material observed in the Kuiper Belt. The colors are more similar to the active cometary nuclei and damocloids. Buratti et al. (2005) show that the color of the dark side of Iapetus is consistent with dust from the small outer satellites of Saturn. Buratti et al. also find that none of Saturn's irregular satellites have similar spectrophotometry as Phoebe. The irregular satellites of Uranus have a wide range of colors from the bluest to the reddest. These satellites may show the extreme red colors observed in the Kuiper Belt and have a distribution similar to the Centaurs. Neptune's irregulars have limited observational data but to date they don't show the extreme red colors seen in the Kuiper Belt.

\subsection{Spectra and Albedos}

Near-Infrared and optical spectra of the brightest Jupiter satellites are mostly linear and featureless (Luu 1991; Brown 2000; Jarvis et al. 2000; Chamberlain \& Brown 2004; Geballe et al. 2002). Jarvis et al. (2000) finds a possible 0.7 micron absorption feature in Jupiter's Himalia and attributes this to oxidized iron in phyllosilicates which is typically 


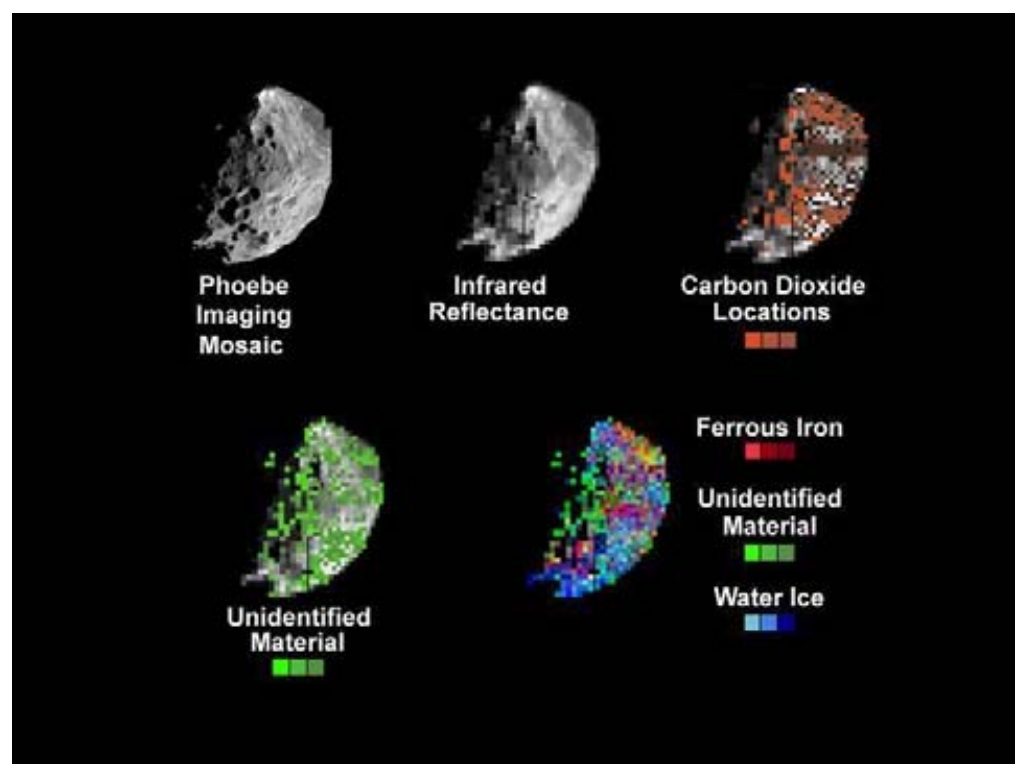

Figure 8. Phoebe's mineral distribution as seen by the Cassini spacecraft. Phoebe appears to have a very volatile rich surface which is unlike the irregular satellites at Jupiter. (Produced by NASA/JPL/University of Arizona/LPL using data from the Cassini Imager and VIMS; see Porco et al. 2005 and Clark et al. 2005).

produced by aqueous alteration. The spectra of Jupiter's irregular satellites are consistent with C-type asteroids. The irregular satellites at Saturn and Neptune appear to be remarkably different with rich volatile surfaces.. The largest Saturn irregular, Phoebe, has been found to have water ice (Owen et al. 1999) as has the large Neptune irregular satellite Nereid (Brown, Koresko \& Blake 1998).

Jupiter's irregular satellites have very low albedos of about 0.04 and 0.05 which again along with their colors are consistent with dark $\mathrm{C}, \mathrm{P}$ and D-type Carbon rich asteroids in the outer main belt (Cruikshank 1977) and very similar to the Jovian Trojans (Fernandez et al. 2003). Saturn's Phoebe has an average albedo of about 0.07 (Simonelli et al. 1999) while Neptune's Nereid was found to have an albedo of 0.16 from Voyager data (Thomas et al. 1991). These albedos are more similar to the higher albedos found in the Kuiper Belt (Grundy et al. 2005; Cruikshank et al. 2005). These are in comparison to the average albedos of comet nuclei 0.03, extinct comets 0.03, and Jovian Trojans 0.06 (Fernandez et al. 2003).

The Cassini spacecraft obtained resolved images of Himalia and showed it to be an elongated shaped object with axes of $150 \times 120 \mathrm{~km}$ with an albedo of about 0.05 (Porco et al. 2003). Cassini obtained a mostly featureless near-infrared spectrum of Jupiter's JVI Himalia (Chamberlain \& Brown 2004).

Cassini obtained much higher resolution images of Saturn's irregular satellite Phoebe (Figure 8) with a flyby of $2071 \mathrm{~km}$ on June 11, 2004. The images showed Phoebe to be intensively cratered with many high albedo patches near crater walls (Porco et al. 2005). Phoebe's density was found to be $1630 \pm 33 \mathrm{~kg} \mathrm{~m}^{-3}$ (Porco et al. 2005). The spectra showed lots of water ice as well as ferrous-iron-bearing minerals, bound water, trapped $\mathrm{CO}_{2}$, phyllosilicates, organics, nitriles and cyanide compounds on the surface (Clark et al. 2005). Phoebe's volatile rich surface and many compounds infer the object was formed beyond the rocky main belt of asteroids and maybe very similar to the composition of comets. Its relatively high density compared to that observed for comets and inferred for 
Kuiper Belt objects makes it a good candidate to have formed near its current location where the highly volatile materials are still unstable to evaporation.

\section{Comparison of the Giant Planet Irregular Satellite Systems}

\subsection{Giant Planet Formation}

Irregular satellites are believed to have been captured around the time of the formation of the giant planets. Thus their dynamical and physical properties are valuable clues as to what happened during the planet formation process. Because of the massive hydrogen and helium envelopes of the gas giants Jupiter and Saturn, they presumably formed quickly in the solar nebula before the gas had time to significantly dissipate. The less massive and deficient in hydrogen and helium ice giants Uranus and Neptune appear to have taken a drastically different route of evolution.

There are two main models for giant planet formation. The standard model of core accretion assumes the cores of the giant planets were formed through oligarchic growth for about $10^{6}$ to $10^{8}$ years. Once they obtained a core of about ten Earth masses they quickly accreted their massive gaseous envelopes (Pollack et al. 1996). The disadvantage of this model is that the protoplanetary disk likely dissipated within a few million years while the core accretion model requires long timescales to form the planets. Because of the lower surface density and larger collisional timescales for more distant planets the core accretion model can not adequately form Uranus and Neptune in the age of the solar system.

The second giant planet formation mechanism is through disk instabilities. This model suggests parts of the solar nebula became unstable to gravitational collapse (Boss 2001). In this model the planets would form on timescales of only about $10^{4}$ years. The disadvantages are it doesn't allow for massive cores and does not appear to be applicable to the small masses of Uranus and Neptune.

Both giant planet formation models have trouble forming Uranus and Neptune (Bodenheimer \& Pollack 1986; Pollack et al. 1996). Any theory on the different formation scenarios of Uranus and Neptune to that of Jupiter and Saturn should take into account the irregular satellite systems of each. The recent theory that Uranus and Neptune lost their hydrogen and helium envelopes by photoevaporation from nearby OB stars (Boss, Wetherill, \& Haghighipour 2002) would have caused all their irregular satellites to be lost because of the significant decrease in the planet's mass (Sheppard and Jewitt (2003); Jewitt \& Sheppard (2005)). Another recent theory is that Uranus and Neptune formed in the Jupiter-Saturn region with subsequent scattering to their current locations (Thommes, Duncan, \& Levison 2002). Any large migration by the planets would have disrupted any outer satellite orbits (Beauge et al. 2002).

\subsection{Population and Size Distributions of the Irregular Satellites}

When measured to a given size the population and size distributions of the irregular satellites of each of the giant planets appears to be very similar (Figure 9) (Sheppard and Jewitt 2005; Jewitt and Sheppard 2005). In order to model the irregular satellite size distribution we use a differential power-law radius distribution of the form $n(r) d r=$ $\Gamma r^{-q} d r$, where $\Gamma$ and $q$ are constants, $r$ is the radius of the satellite, and $n(r) d r$ is the number of satellites with radii in the range $r$ to $r+d r$. All giant planet irregular satellite systems appear to have shallow power law distribution of $q \sim 2$. If we don't include Triton the largest irregular satellite of each planet is of the $150 \mathrm{~km}$ scale with about one hundred irregular satellites expected around each planet with radii larger than about 


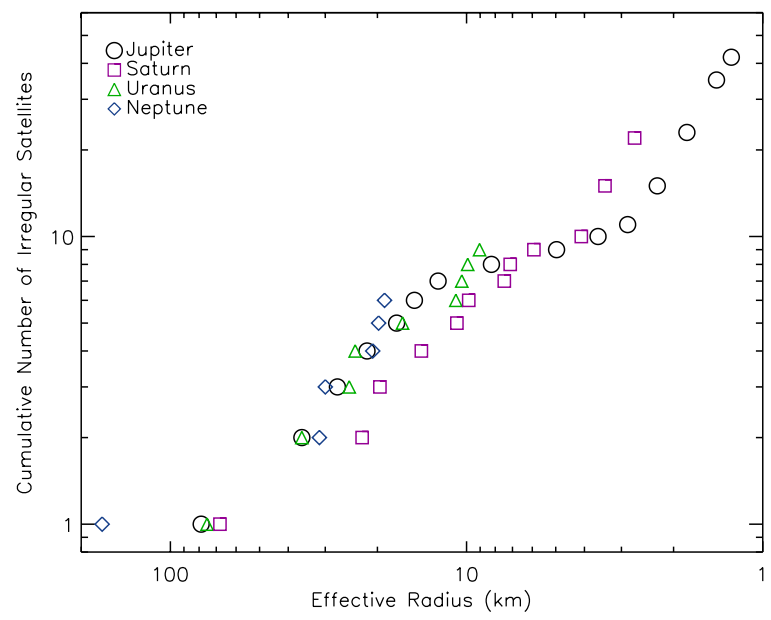

Figure 9. Cumulative radius function for the irregular satellites of Jupiter, Saturn, Uranus and Neptune. This figure directly compares the sizes of the satellites of the giant planets assuming all satellite populations have similar low albedos. The planets have statistically similar shallow size distributions of irregular satellites. Neptune's irregular satellite size distribution is plotted without including Triton. [Modified from Sheppard et al. 2005]

$1 \mathrm{~km}$. This is unexpected considering the different formation scenarios envisioned for the gas giants versus the ice giants.

\section{Discussion and Conclusions}

The irregular satellites of each planet are a distinct group of bodies not necessarily linked to the two prominent reservoirs of the main asteroid belt or the Kuiper Belt. These satellites may have formed relatively near their current locations and were subsequently captured by their respective planet near the end of the planet formation epoch. With the development of large, sensitive, digital detectors on large class telescopes in the late 1990's the discovery and characterization of the irregular satellites improved dramatically. We find that the gas giants Jupiter and Saturn and the ice giants Uranus and Neptune all have a system of irregular satellites which have similar sizes, populations and dynamics.

Current observations favor the capture mechanism of collisional or collisionless interactions within the Hill spheres of the planets. This capture mechanism is fairly independent of the planets formation scenario and mass unlike gas drag or pull-down capture (Jewitt \& Sheppard 2005). Because the less massive ice giants are more distant from the Sun their Hill spheres are actually larger than the gas giants. These increased Hill spheres may compensate for the lower density of small bodies in the outer solar nebula and thus allow all the giant planets to capture similar irregular satellite systems. Recent discoveries of binaries in the Kuiper Belt show that such objects may be quite common in the outer solar system. These binary pairs would be ideal for creating irregular satellites of the giant planets through three body interactions as has been shown for the capture of Triton (Agnor \& Hamilton 2004). In fact, the equally sized binary pairs in the Kuiper Belt may have formed in a similar manner (Funato et al. 2004).

Three body interactions would have been much more probable in the early solar system just after planet formation when leftover debris was still abundant. This capture process would allow for the possible scattering predicted for Uranus and Neptune unlike gas drag and pull-down capture since capture by three body interactions would still operate 
after any scattering. Three body capture also agrees with the results of Beauge et al. (2002) in which they find the irregular satellites would have to have formed after any significant planetary migration as well as Brunini et al. (2002) who find that Uranus' irregular satellites would have to be captured after any impact which would have tilted the planet's rotation axis. Also, Triton may have disrupted the outer satellites of Neptune and capture of these irregulars may have occurred after Triton was captured (Cuk \& Gladman 2005). These scenarios all point to satellite capture happening just after the planet formation process.

If three body interactions were the main capture mechanism then one may expect the terrestrial planets to have irregular satellites. The terrestrial planets had very small Hill spheres compared to the giant planets because of their low mass and proximity to the Sun. In addition, the terrestrial planets had no population of regular satellites for passing objects to possibly interact with. This may explain why Mars and the other terrestrial planets have no outer satellites, though Mars' two inner satellites may have been capture through three body interactions. Perhaps Phobos and/or Deimos were once binary asteroids.

The observed irregular satellite dynamical families were probably created after capture. In order to have a high probability of impact for the creation of families either the captured had to occur very early on when collisions were much more probable than now or there must have been a much larger population of now defunct satellites around each planet.

The non-detection of volatiles on Jupiter's irregular satellites whereas volatiles are seen on Saturn's and Neptune's bodes well for the objects to have formed near their current location. The currently limited data on the albedos, colors and densities of the irregular satellites appear to show that each planet's irregular satellites are physically distinct. Jupiter's irregulars are remarkably similar to the Jovian Trojans and dead comets. Saturn's are significantly redder but neither Jupiter's or Saturn's show the very red material observed in the Kuiper Belt. Uranus' irregulars have a wide range of colors with some being the bluest and others being the reddest.

\section{Acknowledgements}

Support for this work was provided by NASA through Hubble Fellowship grant \# awarded by the Space Telescope Science Institute, which is operated by the Association of Universities for Research in Astronomy, Inc., for NASA, under contract NAS 5-26555.

\section{References}

Agafonova, I. \& Drobyshevski, E. 1985, EMEP 33, 1

Agnor, C. \& Hamilton, D. 2004, BAAS 36, 40.14

Astakhov, S., Burbanks, A., Wiggins, S., \& Farrelly, D. 2003, Nature 423, 264

Barucci, M., Fulchignoni, M., Birlan, M., Doressoundiram, A., Romon, J., \& Boehnhardt, H. 2001, AA 371, 1150

Beauge, C., Roig, F., \& Nesvorny, D. 2002, Icarus 158, 483

Bodenheimer, P. \& Pollack, J. 1986, Icarus 67, 391

Boss, A. 2001, ApJ 563, 367

Boss, A. 2002, ApJ 599, 577

Boss, A., Wetherill, G., \& Haghighipour, N. 2002, Icarus 156, 291

Brown, M., Koresko, C., \& Blake, G. 1998, ApJ 508, 175

Brown, M. 2000, AJ 119, 977

Brunini, A. \& Conicet, P. 1995, EMESP 71, 281

Brunini, A., Parisi, M., \& Tancredi, G. 2002, Icarus 159, 166 
Buratti, B., Hicks, M., \& Davies, A. 2005, Icarus 175, 490

Burns, J. 1986, in Satellites ed. by J. Burns and M. Matthews (University of Arizona Press, Tucson) pg. 117

Burns, J. 1992, in Mars ed. by Kieffer, Jakosky, Snyder, Matthews (University of Arizona Press, Tucson) pg. 1283

Canup, R. \& Asphaug, E. 2001, Nature 412, 708

Carruba, V., Burns, J., Nicholson, P., \& Gladman, B. 2002, Icarus 158, 434

Colombo, G. \& Franklin, F. 1971, Icarus 15, 186

Chamberlain, M. \& Brown, R. 2004, Icarus 172, 163

Clark, R., Brown, R., Jaumann, R. et al. 2005, Nature 435, 66

Cruikshank, D. 1977, Icarus 30, 224

Cruikshank, D., Stansberry, J., Emery, J., Fernandez, Y., Werner, M., Trilling, D., \& Rieke, G. 2005, ApJ 624, 53

Cuk, M. \& Burns, J. 2004, Icarus 167, 369

Cuk, M. \& Gladman, B. 2005, ApJ 626, 113

Dahlgren, M. \& Lagerkvist, C. 1995, AA 302, 907

Degewij, J., Zellner, B., \& Andersson, L. 1980, Icarus 44, 520

Everhart, E. 1973, AJ 78, 316

Fernandez, Y., Sheppard, S., \& Jewitt, D. 2003, AJ 126, 1563

Funato, Y., Makino, J., Hut, P., Kokubo, E., \& Kinoshita, D. 2004, Nature 427, 518

Geballe, T., Dalle, O., Cruikshank, D., \& Owen, T. 2002, Icarus 159, 542

Gladman, B., Nicholson, P., Burns, J., Kavelaars, J., Marsden, B., Williams, G., \& Offutt, W. 1998, Nature 392, 897

Gladman, B., Kavelaars, J., Holman, M., Petit, J., Scholl, H., Nicholson, P., \& Burns, J. 2000, Icarus 147,320

Gladman, B., Kavelaars, J., Holman, M., Nicholson, P., Burns, J. et al. 2001, Nature 412, 163

Goldreich, P., Murray, N., Longaretti, P., \& Banfield, D. 1989, Science 245, 500

Grav, T., Holman, M., Gladman, B., \& Aksnes, K. 2003, Icarus 166, 33

Grav, T., Holman, M., \& Fraser, W. 2004a, ApJ 613, 77

Grav, T. \& Holman, M. 2004b, ApJ 605, 141

Grundy, W., Noll, K., \& Stephens, D. 2005, Icarus 176, 184

Hamilton, D. \& Krivov, A. 1997, Icarus 128, 241

Henon, M. 1970, AA 9, 24

Heppenheimer, T. \& Porco, C. 1977, Icarus 30, 385

Holman, M., Kavelaars, J., Grav, T., Gladman, B. et al. 2004, Nature 430, 865

Hunter, R. 1967, MNRAS 135, 245

Jarvis, K., Vilas, F., Larson, S., \& Gaffey, M. 2000, Icarus 145, 445

Jewitt, D. \& Luu, J. 2001, AJ 122, 2099

Jewitt, D. 2002, $A J$ 123, 1039

Jewitt, D., Sheppard, S., \& Porco, C. 2004, in: F. Bagenal (ed.), Jupiter: the planet, satellites and magnetosphere (Cambridge: Cambridge University Press), p. 263

Jewitt, D. \& Sheppard, S. 2005, Space Sci Rev 116, 441

Jewitt, D. 2005, AJ 129, 530

Kavelaars, J., Holman, M., Grav, T., Milisavljevic, D., Fraser, W., Gladman, B. J., Petit, J.-M., Rousselot, P., Mousis, O., \& Nicholson, P. 2004, Icarus 169, 474

Kessler, D. 1981, Icarus 48, 39

Kowal, C., Aksnes, K., Marsden, B., \& Roemer, E. 1975, AJ 80, 460

Kozai, Y. 1962, AJ 67, 591

Krivov, A., Wardinski, I., Spahn, F., Kruger, H., \& Grun, E. 2002, Icarus 157, 436

Kuiper, G. 1956, Vistas Astron. 2, 1631

Kuiper, G. 1961, in: G.P. Kuiper and B.M. Middlehurst (eds.), Planets and Satellites) (Chicago: University of Chicago Press) p. 575

Luu, J. 1991, AJ 102, 1213

Maris, M., Carraro, G., Cremonese, G., \& Fulle, M. 2001, AJ 121, 2800

Moulton, F. 1914, MNRAS 75, 40 
Nesvorny, D., Alvarellos, J., Dones, L., \& Levison, H. 2003, AJ 126, 398

Nesvorny, D., Beauge, C., \& Dones, L. 2004, AJ 127, 1768

Owen, T., Cruikshank, D., Dalle, O., Geballe, T., Roush, T., \& de Bergh, C. 1999, Icarus 139, 379

Peale, S. 1999, ARA 6 A 37, 533

Peixinho, N., Lacerda, P., Ortiz, J., Doressoundiram, A., Roos-Serote, M., \& Gutierrez, P. 2001, AA 371,753

Pollack, J., Burns, J., \& Tauber, M. 1979, Icarus 37, 587

Pollack, J., Hubickyj, O., Bodenheimer, P., Lissauer, J., Podolak, M., \& Greenzweig, Y. 1996, Icarus 124,62

Porco, C., West, R., McEwen, A. et al. 2003, Sci 229, 1541

Porco, C., Baker, E., Barbara, J. et al. 2005, Sci 307, 1243

Rettig, T., Walsh, K., \& Consolmagno, G. 2001, Icarus 154, 313

Saha, P. \& Tremaine, S. 1993, Icarus 106, 549

Sheppard, S. \& Jewitt, D. 2003, Nature 423, 261

Sheppard, S., Jewitt, D., \& Kleyna, J. 2004, AJ 128, 2542

Sheppard, S., Jewitt, D., \& Kleyna, J. 2005, AJ 129, 518

Sheppard, S., Jewitt, D., \& Kleyna, J. 2006, $A J$ submitted

Simonelli, D., Kay, J., Adinolfi, D., Veverka, J., Thomas, P., \& Helfenstein, P. 1999, Icarus 138, 249

Sykes, M., Cutri, R., Fowler, J., Tholen, D., Skrutskie, M, Price, S., \& Tedesco, E. 2000, Icarus 143,371

Tholen, D. \& Zellner, B. 1984, Icarus 58, 246

Thomas, P., Veverka, J., \& Helfenstein, P. 1991, JGR 96, 19253

Thommes, E., Duncan, M., \& Levison, H. 2002, AJ 123, 2862

Tsui, K. 2000, Icarus 148, 139

Whipple, A. \& Shelus, P. 1993, Icarus 101, 265 